\title{
Network Intrusion Detection Techniques and Network Attack Types
}

\author{
Rajeswari P. V. N., B. Rasagna, K. Sireesha, Sk. Shahina Begum
}

\begin{abstract}
Dynamic: With the huge improvement in innovation and with the big utilization of internet, massive increment in internet dangers has been seeing which activates contriving of latest strategies in device protection. those types of gadget attacks as some distance as unapproved get to, unordinary assaults can be remedy making use of network Intrusion Detection device(NIDS). machine Anomaly Detection(NAD) framework is a particular assortment of IDS. it's miles a corresponding innovation to frameworks that distinguish safety dangers depending on parcel marks. In NAD, the device is unendingly decided for event of anomalous activities or unexpected assaults. by way of utilising this NAD techniques, it is plausible to select out in the occasion that all and sundry tries to attack property or particular has with the aid of using and contrasting and the records accrued from past regarded assaults. This paper gives diagram on severa classifications NID techniques and moreover numerous styles of structures assaults. We bear in mind that this audit will provide a advanced keen of the special commands of attack sorts records, which gives degree to analyze to retain similarly.
\end{abstract}

Watchwords: laptop systems, network safety, community interruption discovery, attacks, dispensed DoS assault.

\section{ADVENT}

With the developmental changes in innovation, and with the presentation of web idea, web become the wellspring of records for an applications. Parallelly, currently a-days it's miles established because the purpose for wonderful virtual assaults. As in keeping with Anderson [1], an interruption challenge or a peril is a practical and unlawful venture to (I) get get entry to on mystery information, (ii) regulate or control the prevailing facts, or (iii) to reason the framework dishonest or not appropriate to anybody. for instance, (a) Denial of issuer (DoS) assault endeavors to be malnourished a large corporation of its assets, which can be required for making ready the data correctly; (b) Worms and infections get gain of greater has through the machine for breaking down; and (c) Compromises acquire unique get right of entry to to a number with the aid of way of taking focal elements of diagnosed vulnerabilities. diverse calculations and techniques are familiar with cozy the system framework and correspondence over the net, among them the

utilization of firewalls idea, one of a type varieties of encryption strategies, and digital private systems are

Revised Version Manuscript Received on August 19, 2019.

Rajeswari P. V. N., Dept of Comp. Sc. \& Engg., Visvodaya Technical Academy, Kavali, AP, India.(email: rajivrphd@gmail.com)

B. Rasagna, Dept of Comp. Sc. \& Engg., Visvodaya Technical Academy, Kavali, AP, India.(email: rasagnabheema87@gmail.com)

K. Sireesha, Dept of Comp. Sc. \& Engg., Visvodaya Technical Academy, Kavali, AP, India.(email: kalapati.sireesha85@gmail.com)

Sk. Shahina Begum, Dept of Comp. Sc. \& Engg., Visvodaya Technical Academy, Kavali, AP, India.(email: shaheena.vits@gmail.com) assuming a essential manner. For execution of NIDS, basically methodologies are applied specifically: signature primarily based and abnormality based completely

vicinity [10]. The primary approach has showed as a business enterprise fulfillment. In mark based totally definitely strategy, NIDS will assist with a variety of marks, in which each considered one in every of which separate the diagram of a fantastic safety danger (for instance an contamination, or a trojan horse or a Denial of provider(DoS) assault). what's more, Anomaly based NIDS constantly check out device site visitors and look at it towards a conventional wellknown of regular site visitors layout. Contingent on this gave modern we are capable of separate what's "ordinary or normal" traffic inside the framework - for example, elegant information transmission use, the numerous conventions applied normally, proper combination of ports numbers for numerous applications in various gadgets. In mild of this distinction, the framework will act both because the pinnacle or the consumer at some thing factor uncommon web site traffic flow into is visible it's extensively divergent as of the benchmark. The method, oddity primarily based interruption reputation in computer structures alludes as an answer for the issue of coming across extraordinary examples in device site visitors that goes amiss from the regular everyday behavior. This form of bizarre examples are usually named as irregularities, anomalies, special instances, distortions, astonishments or attacks. As of now, Anomaly identification has extensively required in a part of makes use of, as an example, extortion discovery in budgetary branch to verify rate playing cards exchanges, interruption acknowledgment in remember or digital safety, and moreover in military supervision to observe the foe activities.

\section{CLASSIFICATION OF NETWORK INTRUSION DETECTION (NID)STRATEGIES}

In fig1, severa styles of NIDS strategies are given.

\subsection{Statistical primarily based strategies}

Factually, an oddity or exception is an exam that is associated with being in component or honestly unessential motion as it isn't made via the stochastic version created and used[3]. by means of the usage of and large, for a measurable model those genuine techniques healthy (for the maximum issue for ordinary conduct) to the appropriate records and upon this information, a mathematical conclusion check is 
hooked up to finish up if any shrouded event or outline has a place with this model or no longer. After the associated take a look at and depending on the were given length, the created examples from the scholarly version with a low possibility are proclaimed as oddities or assaults. it's far possible that one or each parametric and nonparametric techniques are verified as useful for structuring measurable models for abnormality discovery. The parametric structures gauge the parameters from the given statistics by accepting the gaining knowledge of or information degree of the crucial distribution.[4]. what is greater, the non-parametric techniques do no longer by way of and large accept facts of the crucial dispersion [5]. cover[6] is natural calculation for actual Intrusion Detection tool. cowl up is a peculiarity based IDS in laptop structures.

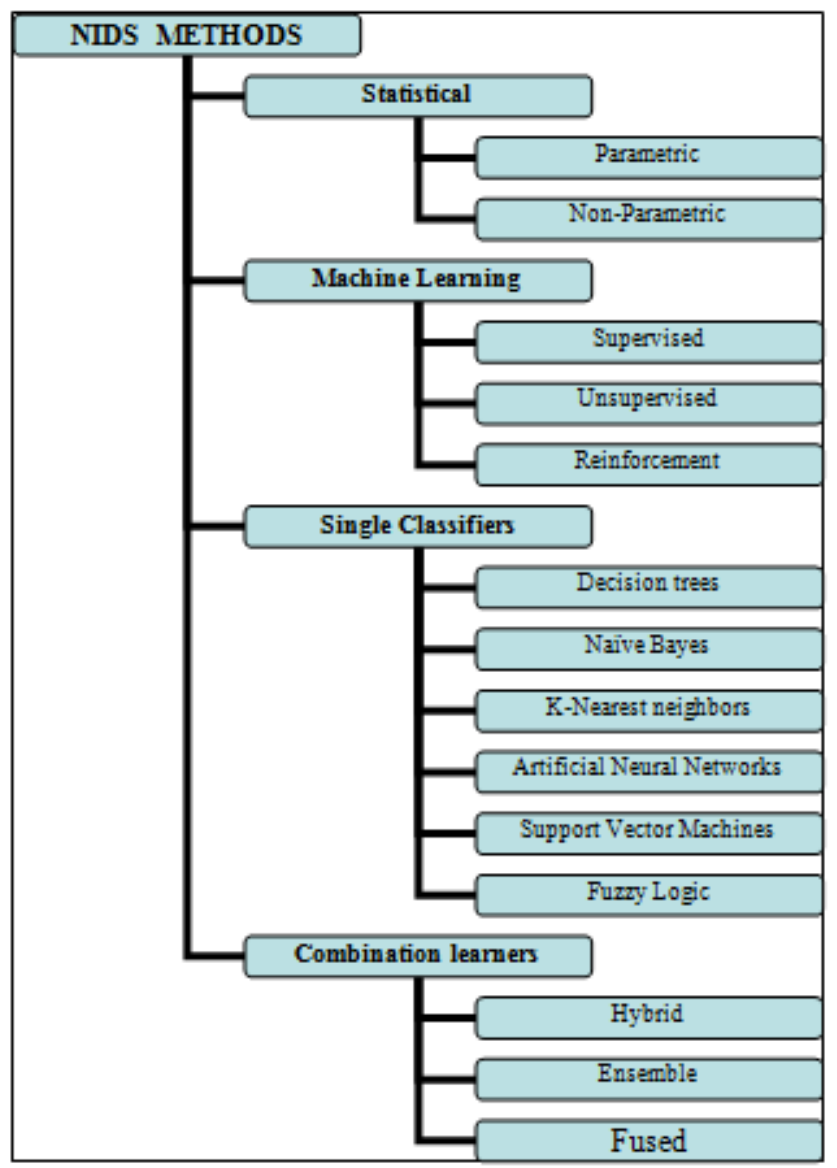

Figure 1:Different types of Network Intrusion Detection techniques.

It makes use of measurable strategies and neural system classifiers to differentiate interruptions. typically, hide is a conveyed framework, which have various ranges in which each degree contains severa Intrusion Detection dealers (IDAs) that are segments of Intrusion Detection gadget(IDS) . the critical thing project of these IDAs is to continuously check the conduct of a number framework or an all out system.

\section{2 device getting to know based totally techniques}

AI may be visible due to the fact the challenge to build laptop obligations or calculations that improvement the exhibition of some task through learning and experience. The actual factor of creating or planning AI programs as to framework or device protection is to decrease the repetitiveness and moreover to lessen the time expended in human assessment studies.

2.2.1 what's gadget gaining knowledge of? In artificial Intelligence(AI), the system gaining knowledge of(ML) proven as a large and massive issue for studies which provide extension to imaginative upgrades in diverse programs or zones and which plans to impressionist realistic abilities of humans through utilizing innovation via machines. inside the system gaining knowledge of studies region, one considers the noteworthy problem this is the manner through manner of which to make machines prepared to "take a look at". in this specific state of affairs, learning is unsaid as inductive stop; in which one investigates fashions that talk to bad.

\subsection{2class of device getting to know}

AI basically ordered as controlled, unaided and Semi-supervised[1] as seemed in figure2.

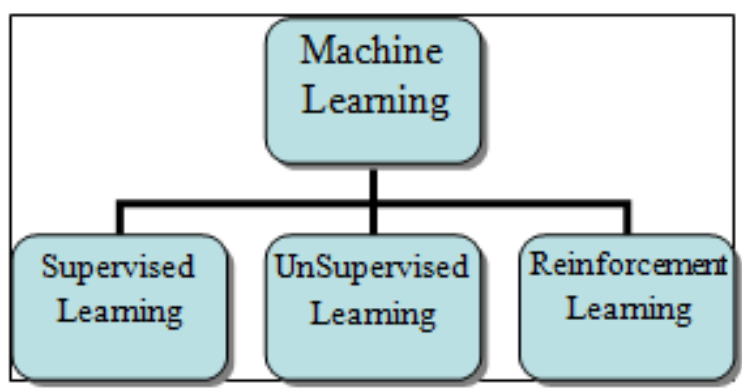

Figure 2: Machine learning techniques

a. Supervised gaining knowledge of This supervised mastering also termed as classification. In supervised learning the information and the times are labeled inside the training phase. There are specific and numerous supervised studying algorithms. among them artificial Neural network(ANN), Bayesian facts with the aid of the use of Bayesian belief Networks(BBN), Gaussian manner methods, Lazy learning, Regression, Nearest Neighbor(NN) set of rules, assist Vector device(SVM), Hidden Markov version(HMM), Bayesian Networks, decision trees(C4.5, ID3, CART, Random Forrest), Hoeffding bounds, okay-nearest neighbor, Boosting, Ensembles classifiers (Bagging, Boosting), Linear Classifiers (Logistic regression, Naive Bayes classifier, Perceptron, Fisher Linear discriminant, SVM), Quadratic classifiers and many others., are various and maximum popular methods in supervised gaining knowledge of algorithms.

b. Un-Supervised gaining knowledge of first of all, in unsupervised mastering, no labels are assigned for generated times. Clustering is considered as a acquainted famous technique for this type of mastering technique. a number of the familiar unsupervised freshmen are Fuzzy clustering, Cluster analysis (ok-means and okay-Mediots), Self-organizing map, Hierarchical clustering, Eclat algorithm, Apriori algorithm, and Outlier detection (nearby outlier element). Monowar, D. k. Bhattacharyya, H. Bhuyan and J. okay. Kalita proposed an set of rules named as TreeClus[28] for finding diverse clusters in available 
community intrusion statistics and to become aware of outliers or mysterious assaults without having any labelled passage or signatures. Juliette Dromard et al proposed every other set of rules termed as ORUNADA[29] with an expansion :online and actual-time Unsupervised network Anomaly Detection set of rules. in this set of rules, the characteristic space is update constantly depending upon a distinct time-sliding windowpane and on incremental grid clustering technique to stumble on the anomalies speedily.

c. Reinforcement mastering the main topic of this Reinforcement gaining knowledge of is, if you want to reap to gain a exact aim, the machine or computer interacts with the surroundings. in this, the reinforcement approach will request the person (e.g., a website professional or area analyst) to provide label to an instance, which can be from a fixed of examples or instances without having any labeling. Arturo Servin and Daniel Kudenko et al proposed technique named as RL-IDS[30], with many community sensor dealers organized in hierarchical structure. in this, by using gazing the local state, each network sensor agent learns to infer the observations, and corresponds them to a central better agent within the supplied agent hierarchy. those central agents will ship the obtained alerts in turn, to the better degrees in hierarchy on demand. As a very last factor, intrusion alarm may be signaled through the agent located on the top of the hierarchy.

\section{2 single Classifiers}

In single classifiers, because it call specifies, simplest one gadget learning set of rules or approach for implementing an intrusion detection gadget can be used as a standalone classifier which is likewise called as unmarried classifier. diverse varieties of information mining standards can be for classification as given underneath:

a. selection Tree the usage of selection Tree(DT), a classifier is evolved for predicting goal elegance price for an unobserved check incidence, based totally on several already recognised examples. via a chain of choices, an unseen take a look at instance is being labeled through a selection tree [7]. decision tree is regularly used as standalone classifier due to its easier illustration and quicker implementation way [11]. selection tree may be prolonged as two distinctive approaches: (i) First one is termed as classification tree, with more than a few representational rank labels and (ii) second one is called as Regression tree, whose magnificence label values as numerical[7].

b. Naive Bayes on this technique, the attributes are provisionally impartial to each other and accordingly attempts to approximate the class-conditional possibility on the supplied class labels[9]. With the existence of less complicated family members, the Naive Bayes frequently produces splendid outcomes within the classification method. It wishes to carry out best one scan of the furnished schooling facts which eases the job of category.

c. okay-nearest neighbor on this, k-nearest neighbor technique, different distance metrics are used. The methodology utilized in that is, at first okay samples are decided on out from the taken schooling set which are closest to the check set and then it allots the most frequent magnificence label among the selected schooling facts to the specified test pattern. This approach is straight forward and nonparametric[2] for classifying samples. okay-nearest neighbor can be referred to for example-primarily based learner, not an inductive based totally [8].

d. synthetic Neural community synthetic Neural community (ANN) method resembles the human brain functionality in processing of data.[12]. commonly neural networks are established in number of layers which are again constructed up with some of interconnected nodes whose functionality managed with activation feature. enter labels or patterns are feed to the neural community through the input layer, which speak to one or extra subsequent connected hidden layers and with a machine of weights for every connection, the actual statistics processing is performed in hidden layer. in addition, the hidden layers are linked to output layer to received the end result using activation feature to proceed the detection method.

a singular technique termed as One-elegance Neural network(OC-NN)[31] became proposed by Raghavendra Chalapathy used in particular to pick out anomalies in complicated facts sets if any. OC-NN method also combines the capability of deep networks to mine gradually high-quality illustration of instances with only one-magnificence with goal of creating boundary limits around ordinary information. that is variation from other techniques which makes use of a hybrid approach in learning deep features, by means of encoding the features after which feeding the functions into a some other approach named as separate anomaly detection approach like one-elegance SVM (OC-SVM).

e. aid Vector Machines a new method changed into introduced in mid-1990's [13] called as aid vector system (SVM). For intrusion detection, the SVM basically treats the education facts as a normal magnificence of gadgets known as non-assault data in intrusion detection device, and thus pretending the ultimate samples as anomalies in the device[14]. the base classifier built through using SVM method discriminates the enter space in a finite vicinity wherein the everyday items are contained and all of the rest of the distance is thought to include the anomalies [15]. Winnipeg, Manitoba [27] anticipated an set of rules which lies on simulated annealing methodology which combines the randomly decided on three distinctive features at a time after which SVM method is applied on that function mixture this is able to hit upon anomalous behavior from the internet facts visitors.

f. Fuzzy common sense this is an advanced idea in reasoning. almost in reasoning, dual common sense's want to be hint i.e., fact values may be either certainly fake (zero) or absolutely authentic (1). In contrast, in Fuzzy logic is comfy with these types of regulations. which means in Fuzzy good judgment, for any given statement, the variety of the degree of reality varies among 0 and 1 in conjunction with 'zero' and '1'[7].

2.3 combination novices on this phase, we gift some techniques and systems which use mixtures of more than one techniques, normally classifiers. 


\section{2.four.1 Hybrid based studying}

A hybrid classifier lets in unique aggregate of more than one device gaining knowledge of algorithms or strategies for decorate the intrusion detection device's overall performance immensely. in this learning, a few clustering-primarily based techniques are used for preprocessing of education samples for doing away with training samples which can be non-representative after which, acquired clustering consequences termed as schooling samples are used for pattern recognition for designing new classifier. for that reason, either supervised or unsupervised mastering techniques are the primary degree of a hybrid classifier [7]. Zurina Mohd Hanapi, Dahlia Asyiqin and Ahmad Zainaddin[32], proposed a brand new technique, in which hybrid or framework experiment was delivered, that's used for NSL dataset to check the steadiness and consistency of the prevailing approach. The end result of precision, take into account and f-cost fee's are as compared with preceding experiment. both dataset covers four principal sorts of assaults, namely Derial of offerings (DoS), user to Root (U2R), far off to neighborhood (R2L) and Probe. acquired results had assured that the hybrid approach finished better detection particularly on little common NSL datatset as compared to novel KDD dataset, through removing redundancy and incomplete elements in the unique dataset

\subsubsection{Ensemble based getting to know}

The vulnerable classifiers performs to some extent higher than a random classifiers. The overall performance of these susceptible classifiers can be improved if multiple weak beginners are combined that is extensively referred to as Ensemble classifier [7]. on this, Ensemble classifier, bagging, boosting and Majority vote are few not unusual techniques for combining multiple vulnerable learners [9]. despite the fact that that the difficulty of the aspect classifiers get gathered inside the ensemble classifier, but it's been turn out as a very efficient overall performance in some mixture. because of this motive, many researchers are showing more interest ensemble classifiers day by day. Nenekazi.N.P. Mkuzangwe turned into developed a new method[33] wherein ok information gain is used as a overall performance sure. This benefit is unique in phrases of applicable capabilities utilized in rising ensemble classifier and it's far acquired via Adaboosting a choice stump that's the vulnerable classifier inside the ensemble.

\section{4.three Fusion based totally learning}

With an growing need of computerized selection making, it's miles critical to improve classification accuracy in comparison to the stand-by myself general selection-based techniques despite the fact that this kind of machine can also have numerous dissimilar data assets. So, a appropriate aggregate of those is the point of interest of the fusion approach[16]. numerous fusion based totally techniques have been carried out for community anomaly detection procedure [17]-[21]. A classification of such strategies is as follows: (i) records degree, (ii) feature level, and (iii) choice degree.

\section{RESULTS \& DISCUSSIONS}

A number of assaults may be detected through modern-day era techniques of NIDS classified the attack types in

numerous approaches. some of these are indexed inside the determine 3 and defined under[22].

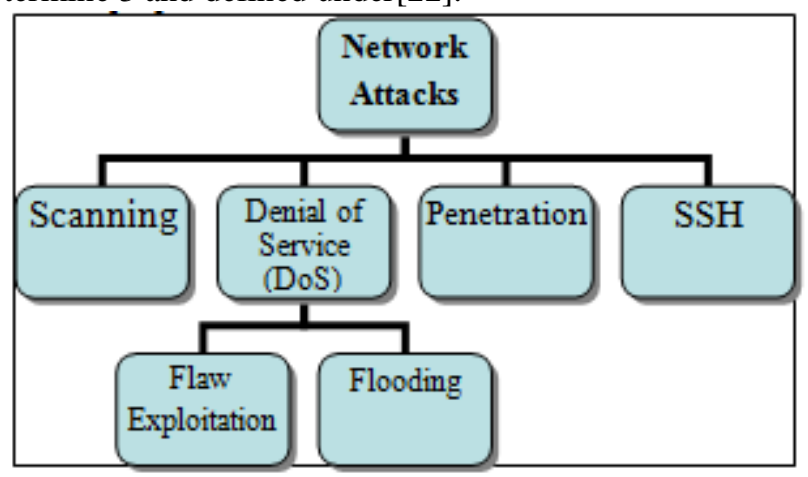

Figure 3: Various NIDS attack types

three.1 Scanning assault

In these form of attacks, an aggressor sends differing styles of statistics bundles to investigate a framework or device for manageable helplessness that can be bullied. on the point while those check bundles are despatched, the goal framework reacts. those reactions are broke right down to decide the peculiarity of the objective framework and to comply with the vulnerabilities assuming any. accordingly filtering attack [23] basically unearths a ability injured individual. machine scanners, port scanners, defenselessness scanners, and so on are applied which surrender these facts. whilst the injured individual is perceived, the assailant can get through them in an unequivocal manner. Filtering all in all taken into consideration as a valid motion and there are a numeral of fashions and programs that use checking method. The famous checking utility is net crawlers. In extra to this, self sustaining individual application assessments a gadget or the complete internet seeking out specific data just, as an example, a tune or video record. A portion of the famous malevolent checking strategies incorporate Vertical and Horizontal port filtering, ICMP vindictive inspecting motion from a actual checking motion with simply high degree of accuracy.(ping) filtering, gradual filter, checking from numerous ports and inspecting of different IP places and ports. NIDS marks may be conceived to distinguish such malevolent filtering action from an real examining action with clearly high stage of precision.

\subsection{Denial of provider(DoS) assault}

With Denial of provider assault, the goal framework will go into reverse or absolutely close down with a purpose to interfere on the administrations and reject the real and permitted customers an entrance in the framework. those attacks are distinctly normal inside the internet where a meeting of hosts are regularly used to attack internet servers with part of phony solicitations. Such types of assaults activates considerable economic damage to internet primarily based enterprise companies with the aid of denying and postponing the customers an anticipated admission to the enterprise. In exceptional, kinds of DoS assaults [24], some of them are pointed out beneath: 


\subsubsection{Flaw Exploitation DoS attacks}

In these hits, an aggressor utilize a defect inside the server programming to both avoid its pace or channel it of guaranteed assets which might be fundamental. Ping of demise assault has a place with this type of assault on a laptop that includes sending a twisted or usually malignant ping to a pc. the dimensions of the ping is commonly 64 bytes (or 84 bytes while IP header is considered). Be that as it could, at whatever point IP bundle length larger than sixty five,535 bytes, the ping cannot deal with in severa laptop frameworks. at the off threat that it tries to send, at that factor it prompts target framework crash. some edges of the conference utilization likewise activates weak spot which may be damaged to execute DoS assaults[6]. The first-rate model for that is, DNS enhancement assault which makes use of ICMP reverberation messages to assault an objective. For these assaults, a mark may be concocted efficiently, for example, to decide a ping of demise assault a NIDS wishes to test the ping banner and parcel size.

three.2.2 Flooding DoS assaults

on this uncommon class of flooding attack, an interloper definitely sends additional solicitations to an objective framework that it can't cope with. this could prompts both burn up of the passing out capability of the goal or channel the objective framework arrange records transmission. With whichever manner of these two will prompts forswearing of management to different administration required clients. DoS attacks are massively tough to war, as those don't abuse any powerlessness within the framework. significantly an increasing number of perilous rendition of DoS attack [5] is known as as dispensed Denial of provider assault (DDoS), which uses an enormous collecting of hosts to focus on a given unlucky casualty have. A programmer that is referred to as as bot ace, can prompt a DDoS attack by utilizing weak spot in a few laptop frameworks, there by get its manage and making this because the DDoS ace for in addition making ready. A brief time later the assailant utilizes this ace to examine with exclusive frameworks (called bots) that can be bargain. whilst a noteworthy quantity of hosts are undermined, at that factor simply with a solitary course, the assailant can start them to begin a scope of flood assaults in opposition to a selected goal.

three. 3 Penetration assault

In front assault [1], an aggressor offers with a framework, and might modify framework nation, study files, compose facts and so forth. For the most element such assaults exploit sure defects within the product, which empowers the aggressor to introduce infections, and malware inside the framework. The most widely identified forms of entrance assaults are:

- person to root: The each section of the framework can gotten to by a close-by patron.

- far off to consumer: in this, a client over the gadget choices up the consumer document and its associated controls absolutely.

- faraway to root: A client over the system offers with the framework.

- far flung plate examine: An aggressor on the machine accesses the tough to attain files put away domestically at the host.

- far off plate compose: An aggressor on the gadget not just accesses the distant facts placed away regionally on the host, however can likewise regulate them.

\subsection{SSH assault}

SSH attacks are a primary quarter of fear for gadget

directors, due to the peril related with an powerful alternate off. The way that the amount of individuals using and relying at the internet is increasing quick makes breaking into and buying and selling off frameworks a perpetually rewarding motion for programmers. One mainstream elegance of attack targets is that of secure Shell ( $\mathrm{SSH}$ ) daemons. by strategies for SSH [23], a programmer can get entry to and likely full authority over faraway hosts. once traded off, a programmer can disrupt the host itself, yet moreover use it for assaulting special frameworks.

\section{2 class Of DDoS assault kinds}

As expressed in [25], a DDoS assault may be specific as successful which makes use of a gigantic range of desktops to start an organized DoS attack adjacent to a solitary or severa unlucky casualty machines. With the purchaser/server innovation, the agent can replica the viability of the DoS attack essentially via saddling the assets of diverse ignorant partner pcs, which fill in as attack stages. it's far established that DDoS assailant is more canny than a DoS aggressor. The numerous types of DDoS attacks are seemed in discern 4[26]. This given characterization depends at the coincidence impact in unfortunate casualties' structures or property. In huge-going, an internet server or middleman server is the real sufferer for a DDoS assault and oversees confined property to offer its management due to these twist of fate, lately arriving bundles want to drop which surpass a few side breaking factors to supervise overabundance machine traffic.. next to losing parcels, it's miles likewise exceeded directly to the senders of the bundles to lower the information stream. valid senders respond for this message by way of diminishing its sending rate. although, the interloper regards this as an accomplishment of its underlying attack execution and improve its rate as a response to the parcel dropping. 


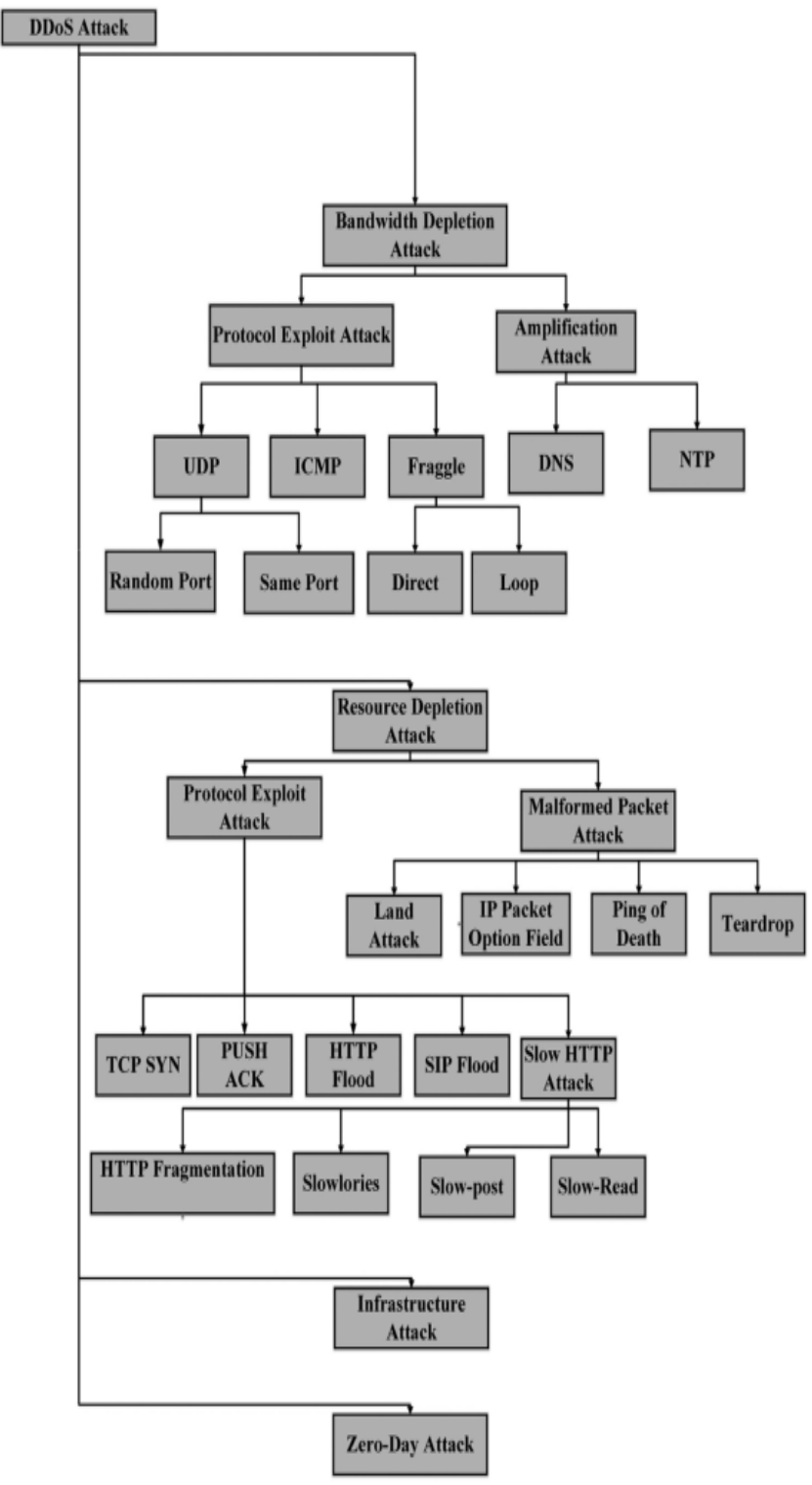

Figure 4: Different categories of DDoS attack types

IlkerOzcelik [33] has gives a area approach on Denial of offerings. This discovery is done via the inconsistency primarily based totally measurements. The Cumulative Sum (Cusum) approach had used to comply with the attack impact at the gadget. This calculation is acting at high and espresso data transmission of the device. The essential aim of this hobby is to represent the higher identity consequences with the cusum calculation because it decreases the misuse of the gadget. Jinghua Yan, Xiaochun Yun proposed a one greater approach for identity of DoS assault[35] making use of weighted outfit version, base classifiers are prepared first using one-of-a-type information characterization calculations (i.e., SVMs, desire tree, and Naive Bayes) on numerous cutting-edge records portions, and after that hundreds every base classifier in keeping with its expectation precision at the modern information. Forestall creating one of a type techniques for identification of interruptions and assaults in structures is giving greater scope for studies in machine understanding which a sub-zone of synthetic perception. on this paper, we offers an define of numerous varieties of NID frameworks and their related calculations. diverse types of security danger of DDoS and severa kinds of distributed assaults likewise cited. at the remaining, we referenced the

Denial of carrier(DDoS) attacks.

\section{REFERENCES}

1. S. Pilleron, D. Sarfati, M.Janssen-Heijnen, J. Vignat, J. Ferlay, F. Bawl, and i.Soerjomataram, "international malignant growth prevalence in more set up grown-ups, 2012 and 2035," A populace-based completely look at. worldwide diary of disorder, July 2018.

2. J. Ferlay, I. Soerjomataram, R. Dikshit, S. Eser, C.Mathers, M. Rebelo, D. M. Parkin, D. Forman, and F. Bawl, "Malignancy incidence and mortality around the arena: property, techniques and massive examples in GLOBOCAN 2012," international diary of sickness, vol. 136, no. 5, pp. E359-E386. 2015

3. R. Thambi, and S. Kandamuthan, "Histopathological evaluation of brain Tumors-A Seven 12 months examine from a Tertiary Care middle in South India," magazine of clinical and indicative research: JCDR, vol. 11, no. 6, pp. EC05. June 2017.

4. M. Lacroix, D. Abi-said, D. R. Fourney ZL. Gokaslan, W. Shi, F. DeMonte, FF Lang, IE. McCutcheon, SJ. Hassenbusch, E. Holland, and k. Hess, "A multivariate exam of 416 patients with glioblastoma multiforme: forecast, degree of resection, and survival," magazine of neurosurgery, vol.90 5, no. 2, pp.a hundred ninety-198. August 2001.

5. N. Sanai, MY. Polley, MW. McDermott, AT. Parsa, and MS. Berger, "A diploma of resection restriction for these days analyzed glioblastomas," journal of neurosurgery, vol. 115, no. 1, pp.three-8. July 2011.

6. MJ. McGirt, D. Mukherjee, KL. Chaichana, KD. Than, JD. Weingart, and A.Quinones-Hinojosa, "courting of exactly procured engine and language deficiencies on with the aid of and massive survival after resection of glioblastoma multiforme," Neurosurgery, vol. sixty 5, no. three, pp. 463-470, SEPTEMBER 2009.

7. C. Kut, KL. Chaichana, J. Xi, SM. Raza, X. Ye, ER McVeigh, FJ. Rodriguez, A. Quiñones-Hinojosa, and X. $\mathrm{Li}$, "Discovery of human cerebrum malignancy invasion ex vivo and in vivo making use of quantitative optical intelligence tomography," technology translational prescription, vol.7, no. 292, pp. 292ra100-292ra100, June 2015.

8. OM. Rygh, T. Selbekk, SH. Torp, S. Lydersen, TAN Hernes, and G. Unsgaard, "examination of explored three-d ultrasound discoveries with histopathology in consequent durations of glioblastoma resection," Acta neurochirurgica, vol. 150, no. 10, p.1033, September 2008.

9. M. Ji, DA. Orringer, CW. Freudiger, S. Ramkissoon, X. Liu, D. Lau, AJ. Golby, I. Norton, M. Hayashi, the huge apple. Agar, and GS. more youthful, "speedy, mark loose identity of cerebrum tumors with energetic Raman dissipating microscopy," science translational remedy, vol. 5, no. 201, pp. 201ra119-201ra119, September 2013.

10. adequate. Özduman, E. Yıldız, A. Dinçer, A. Sav, and MN. Pamir, "making use of intraoperative particular complexity upgraded T1-weighted MRI to apprehend last tumor in glioblastoma scientific system," mag of neurosurgery, vol. a hundred and twenty, no. 1, pp.60-sixty six, January 2014.

11. S .Zausinger, B.cheder, E. Uhl, T. Heigl, D. Morhard, and JC. Tonn, "Intraoperative figured tomography with incorporated path framework in spinal adjustments," backbone, vol. 34, no. 26, pp.2919-2926, 2009. 
12. X. Muñounces, J. Freixenet, X. Cufi, and J. Mart1, "Methodologies for picture department consolidating district and restriction data," sample acknowledgment letters, vol. 24, no. 1, pp.375-392, 2003.

13. S. Krinidis, and V. Chatzis, "A complete of lifestyles fluffy nearby data $\mathrm{C}$-implies grouping calculation," IEEE Transactions on picture Processing, vol.19, no. 5, pp. 1328-1337, can also 2010.

14. J. Shi, and J. Malik, "Standardized cuts and photo department," IEEE Transactions on example research and system insight, vol. 22, no. eight, pp.888-905, August 2000.

15. M. Andersson, J. Gudmundsson, and C. Levcopoulos, "Surmised separation prophets for charts with thick organizations," Lecture notes in software engineering, vol. 3341, pp.fifty three-sixty four. August 2004.

16. G. Govaert, and M. Nadif, "An EM calculation for the rectangular blend version," IEEE Transactions on pattern evaluation and device know-how, vol. 27, no. 4, pp.643-647, April 2005.

17. XF. Wang, and DS. Huang, "A story thickness primarily based grouping form by way of utilising stage set strategy," IEEE Transactions on mastering and facts constructing, vol. 21, no. eleven, pp.1515-1531, information bunching utilising bacterial rummaging development 2009.

18. J. Zhang, and J. Kerekes, "a flexible thickness based totally completely version for isolating ground comes decrease again from photon-checking laser altimeter statistics," IEEE Geoscience and far off Sensing Letters, vol. 12, no. 4, pp.726-730, April 2015.

19. M. Ester, HP Kriegel, J. Sander, and X. Xu, "A thickness based totally totally calculation for finding bunches in huge spatial databases with commotion," In Kdd, vol. ninety six, no. 34, pp. 226-231, August 1996.

20. A. Omrani, and okay. Santhisree, "Bunching consecutive information with OPTICS," In, In lawsuits IEEE 0.33 international convention on communique software program and Networks (ICCSN), pp. 591-594, might also 2011.

21. M. De Marsico, M.Nappi, D. Riccio, and H. Wechsler, "lively face acknowledgment for uncontrolled posture and brightening modifications," IEEE Transactions On systems, guy, And Cybernetics: structures, vol. forty 3, no.1, pp.149-163, 2013.

22. W. Sheng, S. Chen, G. Xiao, J. Mao, and Y. Zheng, "A biometric key age approach relying on semisupervised information grouping," IEEE Transactions on structures, man, and Cybernetics: systems, vol. 45, no. nine, pp.1205-1217, 2015.

23. L. Zhu, FL. Chung, and S.Wang, "Summed up fluffy c-implies grouping calculation with improved fluffy parcels," IEEE Transactions on structures, guy, and Cybernetics, issue B (Cybernetics), vol. 39, no. three, pp.578-591, June 2009.

24. JC. Bezdek, "instance reputation with Fuzzy objective function Algorithms," Kluwer instructional Publishers, Norwell, MA, u.s., 1981.

25. Y. Yang, C. Zheng, and P. Lin, "Fluffy C-implies grouping calculation with a unique punishment term for photograph branch," Optoelectronics assessment, vol. thirteen, no. 4, p.309. 2005.

26. M. Girolami, "Mercer element based bunching in spotlight area," IEEE Transactions on Neural Networks, vol. thirteen, no. 3, pp.780-784, 2002.

27. S. Chen, and D. Zhang, "Hearty picture department making use of FCM with spatial imperatives relying on new aspect initiated separation measure," IEEE Transactions on systems, guy, and Cybernetics, part B (Cybernetics), vol. 34, no. four, pp.1907-1916, August
2004.

28. U. Maulik, and S. Bandyopadhyay, "Hereditary calculation primarily based grouping machine," pattern acknowledgment, vol. 33, no. nine, pp. 455-1465, April 2000.

29. okay. Premalatha, and AM. Natarajan, "a few other technique for information bunching relying on PSO with close by seek," pc and information technology, vol. 1, no. four, p.139, November 2008.

30. M. Wan, L. Li, J. Xiao, C.Wang, and Y Yang, "statistics grouping utilizing bacterial scrounging improvement," magazine of intelligent statistics structures, vol. 38,no. 2, pp.321-341 April 2012.

31. SZ. Selim, and k. Alsultan, "A mimicked tempering calculation for the grouping issue," pattern acknowledgment, vol. 24, no. 10, pp. 1003-1008, January 1991.

32. C. Zhang, D. Ouyang, and J. Ning, "A faux honey bee province technique for grouping," professional systems with programs, vol. 37, no. 7, pp. 4761-4767, July 2010.

33. J. Senthilnath, SN. Omkar, and V. Mani, "Grouping using firefly calculation: execution observe," Swarm and Evolutionary Computation, vol. 1, no. three, pp. 164-171, September 2011.

34. R. Shang, P. Tian, L. Jiao, R. Stolkin, J. Feng, B. Hou, and $\mathrm{X}$. Zhang, "A spatial fluffy bunching calculation with piece metric depending on insusceptible clone for SAR photograph division," IEEE journal of determined on topics in implemented Earth Observations and a ways flung Sensing, vol. 9, no. four, pp. 1640-1652, April 2016.

35. L. Liu, A. Yang, W. Zhou, X. Zhang, M. Fei, and X. Tu, "robust dataset association method relying on neighbor searching and piece fluffy c-implies," IEEE/CAA magazine of Automatica Sinica, vol. 2, no. three, pp. 235-247, July 2015.

36. A. Elazab, C. Wang, F. Jia, J. Wu, G. Li, and Q. Hu, "department of cerebrum tissues from appealing reverberation pics using adaptively regularized piece primarily based fluffy strategies grouping," Computational and clinical strategies in medicine, 2015.

37. YT. Chen, "Medicinal picture Segmentation the usage of impartial issue evaluation-based Kernelized Fuzzy-way Clustering," Mathematical troubles in Engineering, January 2017

38. H. Li, H. He, and Y. Wen, "Dynamic molecule swarm streamlining and okay-implies grouping calculation for picture department," Optik - global journal for mild and Electron Optics, vol. 126, no. 24, pp. 4817-4822, December 2015.

39. H. Ali, M. Elmogy, E. El-Daydamony, and A. Atwan, "Multi-dreams MRI cerebrum image department depending on morphological pyramid and fluffy c-mean bunching," Arabian magazine for era and Engineering, vol.40, no.eleven, pp. 3173-3185, November 2015.

40. E. Abdel-Maksoud, M. Elmogy, and R. Al-Awadi, "Cerebrum tumor department depending on a half breed bunching technique," Egyptian Informatics magazine, vol.16, no.1, pp. seventy one-eighty one, March 2015. 